\title{
Fractional Langevin Equation in Quantum Systems with Memory Effect
}

\author{
Jing-Nuo Wu1 ${ }^{1}$ Hsin-Chien Huang1, Szu-Cheng Cheng1*, Wen-Feng Hsieh ${ }^{2 *}$ \\ ${ }^{1}$ Department of Optoelectric Physics, Chinese Culture University, Taiwan \\ ${ }^{2}$ Department of Photonics and Institute of Electro-Optical Engineering, Taiwan \\ Email: jingnuowu@gmail.com, hchuang@faculty.pccu.edu.tw, \\ wwhsieh@mail.nctu.edu.tw
}

Received 18 April 2014; revised 21 May 2014; accepted 29 May 2014

Copyright (C) 2014 by authors and Scientific Research Publishing Inc.

This work is licensed under the Creative Commons Attribution International License (CC BY).

http://creativecommons.org/licenses/by/4.0/

(c) (i) Open Access

\section{Abstract}

In this paper, we introduce the fractional generalized Langevin equation (FGLE) in quantum systems with memory effect. For a particular form of memory kernel that characterizes the quantum system, we obtain the analytical solution of the FGLE in terms of the two-parameter Mittag-Leffler function. Based on this solution, we study the time evolution of this system including the qubit excited-state energy, polarization and von Neumann entropy. Memory effect of this system is observed directly through the trapping states of these dynamics.

\section{Keywords}

Fractional Generalized Langevin Equation, Memory Effect, Mittag-Leffler Function, Memory Kernel, Trapping States, Polarization, von Neumann Entropy

\section{Introduction}

Application of the fractional calculus on physics has attracted an increasing attention this decade including physical kinetics [1], anomalous transport theory in solid-state physics [2], and nonlinear dynamics [3]. These applications aim to explore the nonlocal quantum phenomena found for either long-range interactions or time-dependent processes with long-time memory effect. Fractional space and time derivatives are used to describe these systems with nonlocal dynamics, e.g. anomalous diffusion or fractional Brownian motion [4] [5]. Recently, the fractional time derivative is given a physical interpretation by Iomin which describes an effective interaction of a quantum system with its environment [6]. Here we will introduce the practical quantum system with long-time memory effect [7].

${ }^{*}$ Corresponding authors. 
Quantum information systems are currently attracting enormous interest for their fundamental nature and potential applications to computation and secure communication. Many novel methods have been proposed to generate controllable qubit states through the environment of the qubits [8]-[10]. When a qubit made of a twolevel atom is embedded inside a structured reservoir with memory effect, the correlation between the qubit and environment will affect the dynamics of the qubit [7] [11] [12]. In this case, the effective interaction of the qubit with the photon modes of the environment is expressed as a memory kernel. In this paper, we take a particular memory kernel of an anisotropic photonic crystal ( $\mathrm{PhC}$ ) as an example to illustrate the method and suitability of applying fractional calculus to this qubit system. We find that the kinetic equation can be expressed as a fractional generalized Langevin equation (FGLE) when the fractional time derivative is used to express memory kernel term. The expression of FGLE opens a new route for the application of fractional calculus to quantum information systems.

This paper is organized as follows. In Section 2, we present a FGLE for the quantum system with memory effect. The general solution of this FGLE is expressed in terms of two-parameter Mittag-Leffler function through the methodology of Laplace transform. In Section 3, a particular memory kernel characterizing the quantum system of a qubit in an anisotropic $\mathrm{PhC}$ is used to illustrate the solving procedures of the FGLE. Based on the analytical solution of this FGLE, we study the dynamics of the qubit energy, polarization and von Neumann entropy. Finally, we summarize our results in Section 4.

\section{Fractional Langevin Equation}

When the quantum system with memory effect is considered, the general form of the kinetic equation can be derived from the time dependent Schrödinger equation as

$$
\frac{\mathrm{d} A(t)}{\mathrm{d} t}=-\int_{0}^{t} K(t-\tau) A(\tau) \mathrm{d} \tau
$$

where $A(t)$ denotes the time evolution of the quantum system and $K(t-\tau)$ the memory kernel of the reservoir. This kinetic equation reads that the future of the system is determined by the memory of the reservoir in its previous state.

To solve Equation (1.1), we introduce the Laplace transform. When the Laplace image of the memory kernel $K(t)$ is considered, we need to construct a Cantor set with infinite number of divisions of time interval because no characteristic time scale exists in this memory kernel. As the Cantor set is constructed, we could choose the entire time interval as $T$ with unity height. When the central part of the time interval is removed, the interval leaves two intervals with length $\xi T(\xi<1 / 2)$. In order to keep the integral memory, the heights of the two intervals must be increased from unity to the value $T /(2 \xi)$. In the next stage, each remaining interval with length $\xi T$ is subjected to the same division process. As the division process is performed $n$ times, the memory kernel in Laplace image will be represented by a set of $2^{n}$ intervals with length $\xi^{n} T$ and height $T /(2 \xi)^{n}$. The integral of the memory kernel in Laplace image is approached as the division time $n$ is taken to be infinity. This approach leads to the Laplace transform of the memory kernel has the form of

$\tilde{K}(s) \equiv \int_{0}^{\infty} \mathrm{e}^{-s t} K(t) \mathrm{d} t \propto(s T)^{-v}$ with $v$ being the fractal dimension of time blocks participating in the construct of the Cantor set.

As the inverse Laplace transform is performed on the kernel $\tilde{K}(s) \cong s^{-v}$, we obtain the memory kernel approximately as

$$
K(t-\tau) \approx(t-\tau)^{v-1} / \Gamma(v)
$$

with Gamma function $\Gamma(x)$. Substituting this memory kernel into Equation (1.1), we have the kinetic equation as

$$
\frac{\mathrm{d} A(t)}{\mathrm{d} t}=-\frac{1}{\Gamma(v)} \int_{0}^{t}(t-\tau)^{v-1} A(\tau) \mathrm{d} \tau .
$$

Comparing the right-hand-side term of this equation with Riemann-Liouville fractional derivative

$$
\frac{\mathrm{d}^{-v} f(t)}{\mathrm{d} t^{-v}}=\frac{1}{\Gamma(v)} \int_{0}^{t}(t-\tau)^{v-1} f(\tau) \mathrm{d} \tau,
$$


we could express the kinetic equation as a differential equation with fractional order, i.e.,

$$
\frac{\mathrm{d} A(t)}{\mathrm{d} t}+\frac{\mathrm{d}^{-v} A(t)}{\mathrm{d} t^{-v}}=0
$$

In order not to lose the initial conditions of the quantum systems, we apply the integral operator $\left(\mathrm{d}^{-1} / \mathrm{d} t^{-1}\right)$ to this equation and obtain

$$
A(t)-A(0)+\frac{\mathrm{d}^{-v-1} A(t)}{\mathrm{d} t^{-v-1}}=0 .
$$

This differential equation with negative fractional order could be further processed through manipulating the fractional differential operator. This manipulation yields

$$
\frac{\mathrm{d}^{v+1} A(t)}{\mathrm{d} t^{\nu+1}}+A(t)=A(0) \frac{t^{-v-1}}{\Gamma(v)} .
$$

Here we have expressed the kinetic equation of this quantum system with memory effect as a FGLE through using the fractional time derivative for the memory kernel.

When we proceed to solve the FGLE through Laplace transform, we need the formula of Laplace transform in fractional order

$$
L\left\{\frac{\mathrm{d}^{\mu}}{\mathrm{d} t^{\mu}} f(t)\right\} \equiv \int_{0}^{\infty} \mathrm{e}^{-s t} \frac{\mathrm{d}^{\mu}}{\mathrm{d} t^{\mu}} f(t) \mathrm{d} t=s^{v} L\{f(t)\}-\sum_{k=0}^{n-1} s^{k}{ }_{0} D_{t}^{\mu-k-1} f(0) \|_{t=0}
$$

with Laplace variable $s$. Here the operator of fractional calculus ${ }_{a} D_{t}^{v}$ is defined as

$$
{ }_{a} D_{t}^{v}= \begin{cases}\mathrm{d}^{v} / \mathrm{d} t^{v}, & \operatorname{Re}[v]>0 \\ 1, & \operatorname{Re}[v]=0 \\ \int_{a}^{t}(\mathrm{~d} \tau)^{-v}, & \operatorname{Re}[v]<0\end{cases}
$$

with $\operatorname{Re}[v]$ being the real part of the order $v$ and the fractional derivative $\mathrm{d}^{v} / \mathrm{d} t^{v}$ being defined through the Riemann-Liouvile form in Equation (1.4). And the Laplace transform of exponential order $t^{-v-1}$ could be obtained from elementary calculus as

$$
L\left[t^{-v-1}\right]=L\left[\frac{\Gamma(-v)}{\Gamma(-v=+n)} \frac{\mathrm{d}^{n}}{\mathrm{~d} t^{n}} t^{-v+n-}\right]=\Gamma(-v) s^{-v-1},
$$

if $-v+n>0$. With these two formula, the Laplace transform of the FGLE has the algebraic form as

$$
\tilde{A}(s)\left[s^{v+1}+1-\left.\left(\sum_{n=0}^{m-1} s^{m-n-1} \frac{\mathrm{d}^{n-m+v+1}}{\mathrm{~d} t^{n-m+v+1}} A(0)\right)\right|_{t=0}\right]=\Gamma(-v) s^{-v-1} .
$$

This algebraic form could be expressed as a sum of partial fractions as

$$
\tilde{A}(s)=\sum_{n} \frac{a_{n}}{\left(s^{v}-X_{n}\right)^{\mu}}
$$

with expansion coefficients $a_{n}$ and $X_{n}$ being the roots of the indicial Equation (1.11).

When the inverse Laplace transform is performed on these fractional expansions of $\tilde{A}(s)$, we need the formula

$$
L^{-1}\left\{\frac{1}{\left(s^{v}-X_{n}\right)}\right\}=\sum_{j=1}^{q} X_{n}^{j-1} E_{t}\left(j v-1, X_{n}^{q}\right)
$$

with positive integer $q=1 / v$ and the two-parameter Mittag-Leffler function $E_{t}(\alpha, a)$. The analytical solution of the FGLE is obtained as 


$$
A(t)=\sum_{n} a_{n} \sum_{j=1}^{q} X_{n}^{j-1} E_{t}\left(j v-1, X_{n}^{q}\right)
$$

which is a linear combination of the two-parameter Mittag-Leffler functions. These two-parameter MittagLeffler functions are defined as

$$
E_{t}(\alpha, a)=\frac{\mathrm{d}^{-\alpha}}{\mathrm{d} t^{-\alpha}} \mathrm{e}^{a t}=t^{\alpha} \sum_{n=0}^{\infty} \frac{(a t)^{n}}{\Gamma(\alpha+n+1)}
$$

with the derivative formula

$$
\frac{\mathrm{d}^{\mu}}{\mathrm{d} t^{\mu}} E_{t}(\alpha, a)=E_{t}(\alpha-\mu, a)
$$

They are related to the one-parameter Mittag-Leffler function $E_{\alpha}(t)=\sum_{n=0}^{\infty} \frac{t^{n}}{\Gamma(1+n \alpha)}$ through $E_{\alpha}\left(a t^{\alpha}\right)=\sum_{n=0}^{q-1} a^{n} E_{t}\left(n \alpha, a^{q}\right)$ with positive integer $q=1 / \alpha \quad[4]$.

\section{A Particular Memory Kernel}

In this section, we consider a quantum system of a qubit in an anisotropic photonic crystal (PhC) with a particular memory kernel as shown in Figure 1. The Hamiltonian of this quantum system is

$$
H=\hbar \omega_{10} \sigma_{11}+\sum_{\vec{k}} \hbar \omega_{\vec{k}} a_{\vec{k}}^{+} a_{\vec{k}}+i \hbar \sum_{\vec{k}} g_{\vec{k}}\left(a_{\vec{k}}^{+} \sigma_{01}-\sigma_{10} a_{\vec{k}}\right) \text {. }
$$

Here the atomic operators $\sigma_{i j}=|i\rangle\langle j|(i, j=0,1)$ obey the commutation relation of $\left[\sigma_{i j}, \sigma_{l m}\right]=\delta_{j l} \sigma_{l m}-\delta_{i m} \sigma_{l j}$ with the Kronecker delta function $\delta_{i j}$. And the photon operators $a_{\vec{k}}$ and $a_{\vec{k}}^{+}$follow the commutation rules of $\left[a_{\vec{k}}, a_{\overrightarrow{k^{\prime}}}\right]=0,\left[a_{\vec{k}}^{+}, a_{\vec{k}^{\prime}}^{+}\right]=0$ and $\left[a_{\vec{k}}, a_{\overrightarrow{k^{\prime}}}^{+}\right]=\delta_{\vec{k} \bar{k}^{\prime}}$. The frequency $\omega_{10}$ stands for the atomic transition frequency from excited state $|1\rangle$ to ground state $|0\rangle$ and $\omega_{\vec{k}}$ for the photon mode frequency of the reservoir with wavevector $\overrightarrow{\boldsymbol{k}}$. The coupling strength between the atom and the photon (electromagnetic field) is characterized by $g_{\vec{k}}=\frac{\omega_{10} \mathrm{~d}_{10}}{\hbar}\left[\frac{\hbar}{2 \varepsilon_{0} \omega_{\vec{k}} V}\right]^{\frac{1}{2}} \hat{\mathrm{e}}_{\vec{k}} \cdot \hat{u}_{\mathrm{d}}$ with the fixed qubit dipole moment $\boldsymbol{d}_{10}=\mathrm{d}_{10} \hat{u}_{d}$, sample volume $V$, dielectric constant $\epsilon_{0}$ and polarization unit vector $\hat{e}_{\vec{k}}$ of the photon mode with frequency $\omega_{\vec{k}}$.

(a)

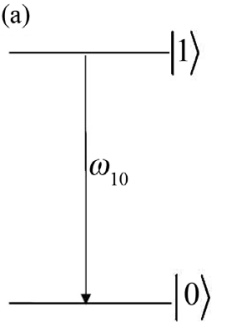

(b)

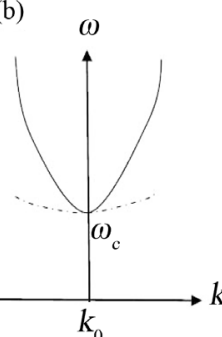

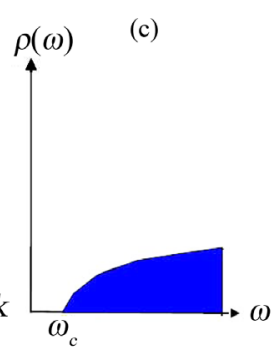

Figure 1. (Color online) (a) A qubit with excited state $|1\rangle$ and ground state $|0\rangle$. The transition frequency $\omega_{10}$ is nearly resonant with the frequency range of the $\mathrm{PhC}$ reservoir; (b) Directional dependent dispersion relations near band edge expressed by the effective-mass approximation with the edge frequency $\omega_{c}$ by solid and dashed curves; (c) Photon DOS $\rho(\omega)$ of the anisotropic PhC reservoir exhibiting forbidden photon mode below the edge frequency $\omega_{c}$. 
As we assume only one photon is created or annihilated for one atomic transition (single-photon sector), the wave function of the system has the form

$$
|\psi(t)\rangle=A(t) \mathrm{e}^{-i \omega_{10} t}\left|1,\left\{0_{\vec{k}}\right\}\right\rangle+D(t)\left|0,\left\{0_{\vec{k}}\right\}\right\rangle+\sum_{\vec{k}} B_{\vec{k}}(t) \mathrm{e}^{-i \omega_{\vec{k}} t}\left|0,\left\{1_{\vec{k}}\right\}\right\rangle
$$

with the initial condition $A(0)=\mathrm{e}^{i \phi_{0}} \cos \left(\frac{\theta_{0}}{2}\right), D(0)=\sin \left(\frac{\theta_{0}}{2}\right)$ and $B_{\vec{k}}(0)=0$. The equations of motion for the amplitudes $A(t), D(t)$ and $B_{\overrightarrow{\boldsymbol{k}}}(t)$ can be obtained when we project the time-dependent Schrődinger equation $H|\psi(t)\rangle=i \hbar \frac{\mathrm{d}|\psi(t)\rangle}{\mathrm{d} t}$ on the single-photon sector of the Hilbert space as

$$
\begin{aligned}
& \frac{\mathrm{d}}{\mathrm{d} t} A(t)=-\sum_{\vec{k}} g_{\vec{k}} B_{\vec{k}}(t) \mathrm{e}^{-i \Omega_{\vec{k}} t}, \\
& \frac{\mathrm{d}}{\mathrm{d} t} B_{\vec{k}}(t)=g_{\vec{k}} A(t) \mathrm{e}^{i \Omega_{\vec{k}} t},
\end{aligned}
$$

and

$$
\frac{\mathrm{d}}{\mathrm{d} t} D(t)=0
$$

with detuning frequency $\Omega_{\vec{k}}=\omega_{\vec{k}}-\omega_{10}$. The two Equations of (1.19) and (1.20) can be combined as

$$
\frac{\mathrm{d}}{\mathrm{d} t} A(t)=-\int_{0}^{t} K(t-\tau) A(\tau) \mathrm{d} \tau
$$

with the memory kernel $K(t-\tau)=\sum_{\vec{k}} g_{\vec{k}}^{2} \mathrm{e}^{-i \Sigma_{\vec{k}}(t-\tau)}$. This evolution equation relates the excited amplitude $A(t)$ of the qubit to the reservoir memory through the memory kernel $K(t-\tau)$. For the anisotropic PhC reservoir, this memory kernel has a particular form of

$$
K(t-\tau)=\frac{\beta^{1 / 2}}{\sqrt{\pi}(t-\tau)^{3 / 2}} \mathrm{e}^{-i[3 \pi / 4-\Delta(t-\tau)]}
$$

with the coupling constant $\beta^{1 / 2}=\left(\omega_{10}^{2} \mathrm{~d}_{10}^{2} \sqrt{\omega_{c}}\right) /\left(16 \pi \epsilon_{0} \hbar c^{3}\right)$ and the detuning frequency $\Delta=\omega_{10}-\omega_{c}$ of the qubit transition frequency $\omega_{10}$ from the band edge frequency $\omega_{c}$ [7]. With this special form of memory kernel, the kinetic equation of the excited amplitude $A(t)$ becomes

$$
\frac{\mathrm{d}}{\mathrm{d} t} C(t)+i \Delta C(t)=\frac{\beta^{1 / 2} \mathrm{e}^{i \pi / 4}}{\sqrt{\pi}} \int_{0}^{t} \frac{C(\tau)}{(t-\tau)^{3 / 2}} \mathrm{~d} \tau
$$

if the transformation $A(t)=\mathrm{e}^{i \Delta t} C(t)$ is performed. Here we express this memory effect as a fractional time derivative which describes the effective interaction of the qubit with the environmental $\mathrm{PhC}$ reservoir. That is, the right-hand-side term of the kinetic Equation (1.24) is written as a Riemann-Louville fractional time derivative with order $v=1 / 2$ and $n=1$ such that

$$
\int_{0}^{t} \frac{C(\tau)}{(t-\tau)^{3 / 2}} \mathrm{~d} \tau=\frac{\Gamma(1 / 2)}{(-1 / 2)} \frac{\mathrm{d}^{1 / 2} C(t)}{\mathrm{d} t^{1 / 2}} .
$$

This expression leads to the fractional form of the kinetic equation as

$$
\frac{\mathrm{d}^{1 / 2}}{\mathrm{~d} t^{1 / 2}} C(t)+i \Delta \frac{\mathrm{d}^{-1 / 2}}{\mathrm{~d} t^{-1 / 2}} C(t)+2 \beta^{1 / 2} \mathrm{e}^{i \pi / 4} C(t)=\frac{t^{-1 / 2}}{\sqrt{\pi}} .
$$

We define this fractional differential equation as a fractional generalized Langevin equation (FGLE) of this quantum system. We solve this FGLE by applying Laplace transform and obtain

$$
\tilde{C}(s)=\frac{1}{s+i \Delta+2 \beta^{1 / 2} \mathrm{e}^{i \pi / 4} s^{1 / 2}} .
$$


Here $\tilde{C}(s)$ is the Laplace transform of $C(t)$ with the initial condition $C(0)=A(0)=1$. In order to find the solution of this excited amplitude, standard procedures of expressing this algebraic equation as a sum of partial fractions and performing inverse Laplace transform on these partial fractions are taken. In the first step, we need to find the roots of the indicial equation $Y^{2}+2 \beta^{1 / 2} \mathrm{e}^{i \pi / 4} Y+i \Delta=0$, where the variable $s^{1 / 2}$ has been converted into $Y$. Two kinds of roots exist in this indicial equation: one with different roots $Y_{1} \neq Y_{2}$ and the other with degenerate root $Y_{1}=Y_{2}$. For the case of different roots, $\tilde{C}(s)$ is expressed as

$$
\tilde{C}(s)=\left[\frac{1}{\left(\sqrt{s}-Y_{1}\right)}-\frac{1}{(\sqrt{s}-Y)}\right] \frac{1}{\left(Y_{1}-Y_{2}\right)}
$$

with

$$
Y_{1}=\mathrm{e}^{i \pi / 4}\left(-\beta^{1 / 2}+\sqrt{\beta-\Delta}\right)
$$

and

$$
Y_{2}=\mathrm{e}^{i \pi / 4}\left(-\beta^{1 / 2}-\sqrt{\beta-\Delta}\right)
$$

For the degenerate case, we have $\beta=\Delta$ which leads to the indicial equation as $\left(Y+\beta^{1 / 2} \mathrm{e}^{i \pi / 4}\right)^{2}=0$. The partial fractions of $\tilde{C}(s)$ is thus written as

$$
\tilde{C}(s)=\frac{1}{\left(\sqrt{s}+\beta^{1 / 2} \mathrm{e}^{i \pi / 4}\right)^{2}} .
$$

As the inverse Laplace transform is applied to these partial-fractional forms of $\tilde{C}(s)$ with fractional powers of the variables $s$, we use the formulas of

$$
L^{-1}\left\{\frac{1}{(\sqrt{s}-a)}\right\}=E_{t}\left(-\frac{1}{2}, a^{2}\right)+a E_{t}\left(0, a^{2}\right)
$$

and

$$
L^{-1}\left\{\frac{1}{(\sqrt{s}-a)^{2}}\right\}=2 a t E_{t}\left(-\frac{1}{2}, a^{2}\right)+a E_{t}\left(\frac{1}{2}, a^{2}\right)+\left(1+2 a^{2} t\right) E_{t}(0, a)
$$

with the fractional exponential function $E_{t}(\alpha, a)$, whose definition and properties are listed in Equations (1.15) and (1.16). This procedure leads to the solution of the fractional kinetic equation being expressed as the linear combination of the fractional exponential functions such that

$$
C(t)=\frac{\mathrm{e}^{-i \pi / 4}}{2 \sqrt{\beta-\Delta}} \times\left[E_{t}\left(-\frac{1}{2}, Y_{1}^{2}\right)-E_{t}\left(-\frac{1}{2}, Y_{2}^{2}\right)+Y_{1} E_{t}\left(0, Y_{1}^{2}\right)-Y_{2} E_{t}\left(0, Y_{2}^{2}\right)\right]
$$

for the different-root case $\beta \neq \Delta$; and

$$
C(t)=(1+2 i t \beta) E_{t}(0, i \beta)-\beta^{1 / 2} \mathrm{e}^{i \pi / 4} E_{t}\left(\frac{1}{2}, i \beta\right)-2 \beta^{3 / 2} \mathrm{e}^{i 3 \pi / 4} E_{t}\left(-\frac{1}{2}, i \beta\right)
$$

for the degenerate case $\beta=\Delta$. Based on this analytic solution, we can obtain the reduced density matrix of the qubit from the wave function in Equation (1.18) through tracing over the reservoir degrees of freedom as

$$
\hat{\rho}_{q}(t) \equiv\left(\begin{array}{ll}
\rho_{11}(t) & \rho_{10}(t) \\
\rho_{01}(t) & \rho_{00}(t)
\end{array}\right)=\left(\begin{array}{ll}
|A(t)|^{2} \cos ^{2}\left(\frac{\theta_{0}}{2}\right) & \frac{1}{2} A^{*}(t) \mathrm{e}^{-i \phi_{0}} \sin \left(\theta_{0}\right) \\
\frac{1}{2} A(t) \mathrm{e}^{i \phi_{0}} \sin \left(\theta_{0}\right) & 1-|A(t)|^{2} \cos ^{2}\left(\frac{\theta_{0}}{2}\right)
\end{array}\right) .
$$


The elements in this matrix are associated with the information of the qubit energy and coherence. In the following, we will study the dynamics of the excited-state energy, polarization and von Neumann entropy of the qubit.

\subsection{Excited-State Probability}

We show the time evolution of the qubit excited-state energy through the probability $P(t)=\rho_{11}(t)=|A(t)|^{2}$ in Figure 2. The energy dynamics exhibits oscillatory behavior and does not decay with time as the qubit frequency lies inside the photonic bang gap (PBG) region $(\Delta / \beta<0)$. The memory effect of this quantum system is observed directly through these trapping states of the qubit energy.

\subsection{Polarization Dynamics}

As a qubit interacts with the environment, it will randomize the polarization of the qubit. The quantum phase information of a qubit carried by the qubit polarization will thus escape from the qubit into the environment through this randomization of polarization and lead to the quantum decoherence. Here we show the polarization dynamics in Figure 3 through the expression of qubit polarization $P_{Z}(t)=\rho_{10}(t)+\rho_{01}(t)=\operatorname{Re}\left[U_{p}(t)\right]$ (if $\left.\phi_{0}=0, \theta_{0}=\pi / 2\right)$. The polarization dynamics of the qubit with frequency lying inside the PBG region $(\Delta / \beta<0)$ exhibits non-decaying oscillation. The qubit loses partial of its polarization in the very beginning period of time and then preserves the remaining polarization through the steady oscillation. This trapping state of the qubit polarization reveals the memory effect of the system which leads to the preservation of the qubit phase information.

\subsection{Dynamics of von Neumann Entropy}

Entropy, a measurement of information amount stored in a qubit, will be changed as the qubit is correlated to the environment. The correlation between the environment and the state will transform the initially pure state of the qubit into a finally mixed state where the amount of information of the qubit is changed. For a qubit state with density matrix $\hat{\rho}(t)$, von Neumann entropy is defined as $S(t)=-\operatorname{Tr}[\hat{\rho}(t) \log \hat{\rho}(t)]=-\sum_{i} \lambda_{i} \log \lambda_{i}$ with $\lambda_{i}$ being the eigenvalues of the matrix $\hat{\rho}(t)$. for the density matrix in Equation (1.36), we show the von Neumann entropy in Figure 4 for the initially excited qubit $\left(\theta_{0}=0\right)$. The entropy has its minimal value zero at $t=0$ and reaches its maximal value $\log 2=0.693$ at the very beginning of time. After a period of time on the order of the decay timescale, the entropy becomes steady with nonzero value for the qubit frequency in PBG region $(\Delta / \beta<0)$. This result shows that the initially pure system becomes maximally mixed in the very beginning period of time. As the qubit equilibrating with the $\mathrm{PhC}$ reservoir, the system becomes steady with less mixed state. The trapping state of the von Neumann entropy reveals that the amount of information stored in the qubit

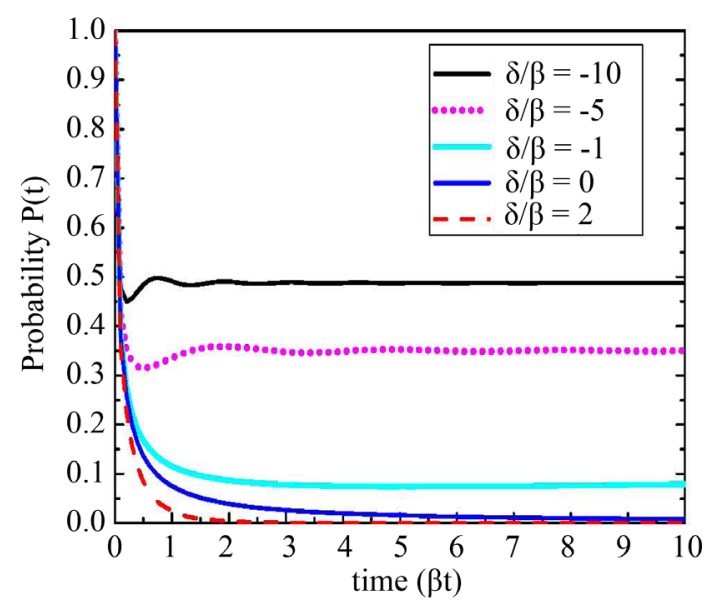

Figure 2. (Color online) Dynamics of the qubit excitedstate probability with different detuning frequencies $\delta / \beta=\left(\omega_{10} / \beta\right)$ from the band edge frequency $\omega_{c}$. 


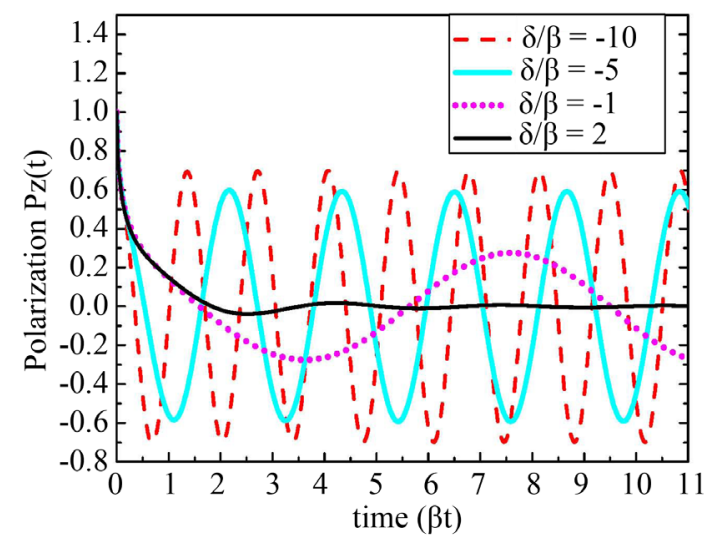

Figure 3. (Color online) Dynamics of the qubit polarization for the qubit frequency lying inside $(\delta / \beta<0)$ and outside $(\delta / \beta>0)$ the band gap.

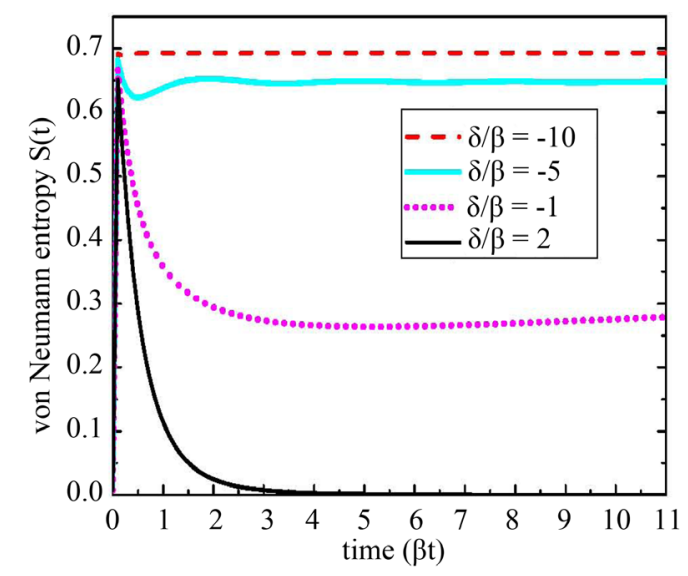

Figure 4. (Color online) Dynamics of the von Neumann entropy for different qubit frequencies.

is preserved through the steady mixed state. The memory effect of the system is observed directly through the preservation of the qubit information.

\section{Conclusion}

We have used the fractional time derivative to express the kinetic equation of the quantum system with memory effect as a FGLE. For a particular memory kernel, we obtain the solution of the FGLE in terms of the twoparameter Mittag-Leffler function. In the study of the qubit dynamics in the particular memory kernel of an anisotropic PhC, we observe the memory effect directly through the trapping states of the qubit energy, polarization and von Neumann entropy.

\section{Acknowledgements}

We would like to gratefully acknowledge partially financial support from the National Science Council (NSC), Taiwan under Contract Nos. NSC 102-2811-M-034-002, NSC 102-2112-M-034-001-MY3 and NSC 102-2112M-009-016-MY3.

\section{References}

[1] Zaslavsky, G.M. (2005) Hamiltonian Chaos and Fractional Dynamics. Oxford University Press, Oxford. 
[2] Metzler, R. and Klafter, J. (2000) The Random Walks Guide to Anomalous Diffusion: A Fractional Dynamics Approach. Physics Reports, 339, 1-77. http://dx.doi.org/10.1016/S0370-1573(00)00070-3

[3] Zaslavsky, G.M. (2002) Chaos, Fractional Kinetics, and Anomalous Transport. Physics Reports, 371, 461-580. http://dx.doi.org/10.1016/S0370-1573(02)00331-9

[4] Miller, K.S. and Ross, B. (1993) An Introduction to the Fractional Calculus and Fractional Differential Equations. John Wiley and Sons, Inc., Hoboken.

[5] Samko, S.G., Kilbas, A.A. and Marichev, O.I. (1993) Fractional Integrals and Derivatives: Theory and Applications. Gordon and Breach, London.

[6] Iomin, A. (2009) Fractional-Time Quantum Dynamics. Physical Review E, 80, Article ID: 022103. http://dx.doi.org/10.1103/PhysRevE.80.022103

[7] Wu, J.-N., Huang, C.-H., Cheng, S.-C. and Hsieh, W.-F. (2010) Spontaneous Emission from a Two-Level Atom in Anisotropic One-Band Photonic Crystals: A Fractional Calculus Approach. Physical Review A, 81, Article ID: 023827. http://dx.doi.org/10.1103/PhysRevA.81.023827

[8] Mazzola, L., Maniscalco, S., Piilo, J., Suominen, K.-A. and Garraway, B.M. (2009) Sudden Death and Sudden Birth of Entanglement in Common Structured Reservoirs. Physical Review A, 79, Article ID: 042302. http://dx.doi.org/10.1103/PhysRevA.79.042302

[9] Maniscalco, S. and Francica, F., Zaffino, R.L., Lo, Gullo, N. and Plastina, F. (2008) Protecting Entanglement via the Quantum Zeno Effect. Physical Review Letters, 100, Article ID: 090503. http://dx.doi.org/10.1103/PhysRevLett.100.090503

[10] Bellomo, B., Lo Franco, R., Maniscalco, S. and Compagno, G. (2008) Entanglement Trapping in Structured Environments. Physical Review A, 78, Article ID: 060302(R).

[11] Jorgensen, M.R., Galusha, J.W. and Bartl, M.H. (2011) Strongly Modified Spontaneous Emission Rates in Diamond-Structured Photonic Crystals. Physical Review Letters, 107, 143902.1-143902.4.

[12] John, S. and Quang, T. (1994) Spontaneous Emission near the Edge of a Photonic Band Gap. Physical Review A, 50, 1764. http://dx.doi.org/10.1103/PhysRevA.50.1764 
Scientific Research Publishing (SCIRP) is one of the largest Open Access journal publishers. It is currently publishing more than 200 open access, online, peer-reviewed journals covering a wide range of academic disciplines. SCIRP serves the worldwide academic communities and contributes to the progress and application of science with its publication.

Other selected journals from SCIRP are listed as below. Submit your manuscript to us via either submit@scirp.org or Online Submission Portal.
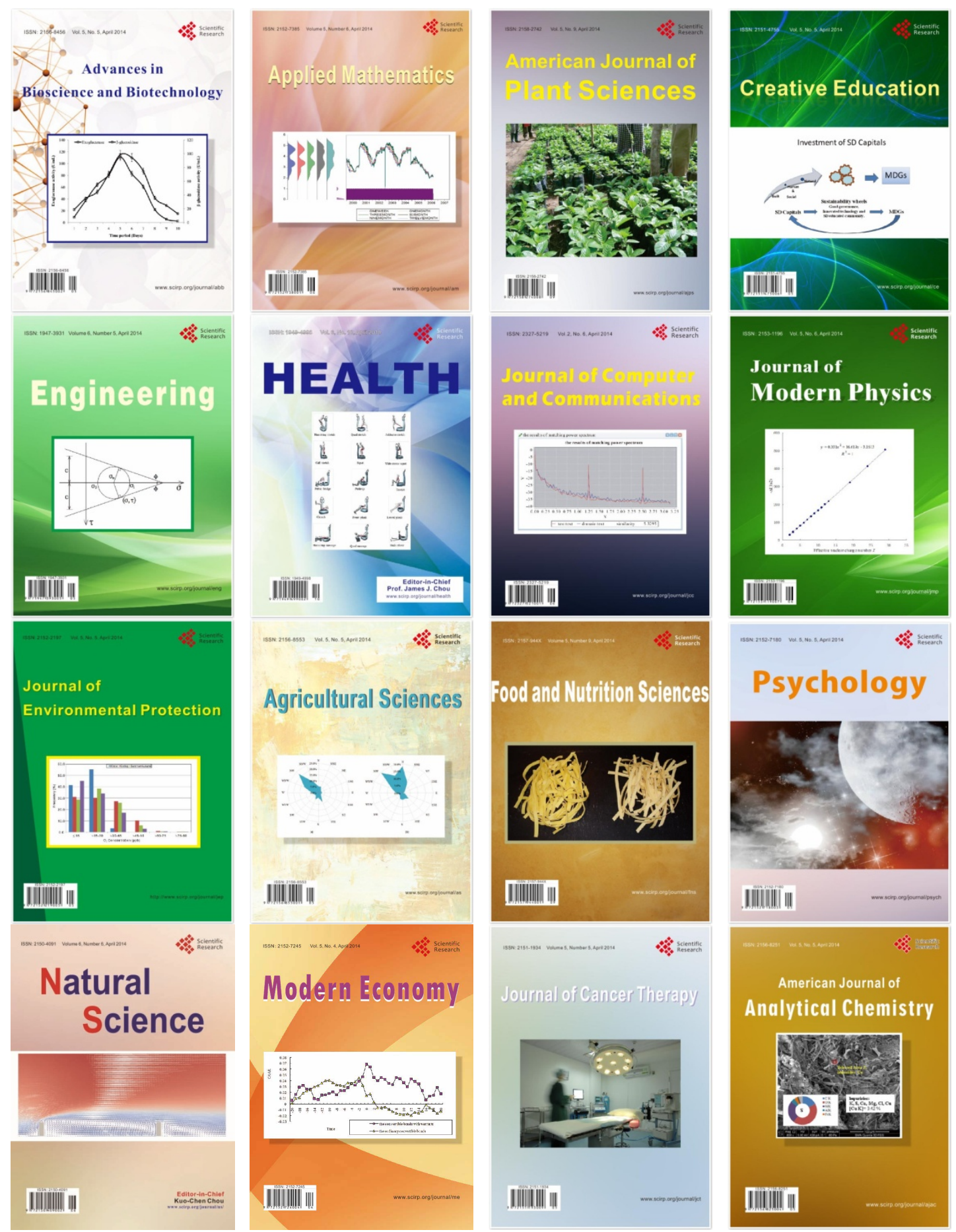